\title{
Zero-energy SIMS depth profiling: The role of surface roughness development with $\mathrm{XeF}_{2}$-based etching
}

\author{
N. Vanhove, ${ }^{a, b *}$ P. Lieven $s^{c}$ and W. Vandervorst ${ }^{a, b}$
}

To obtain monolayer depth resolution in the zero-energy SIMS concept, an almost atomically flat surface during the electron beam-induced etching (EBIE) is crucial. However, the surface roughness observed with Si EBIE and XeF 2 shows the same characteristics as observed during spontaneous etching of $\mathrm{Si}$ with $\mathrm{XeF}_{2}$. The subsurface fluorine concentration in a Si EBIE process (even in a mass transport limited regime) can influence the unactivated formation of the SiF $F_{\mathrm{x}}$ reaction layer as well, and therefore, contribute to observed roughness development. $\mathrm{SiO}_{2}$ EBIE leads to extreme pit formation due to preferential etching of $\mathrm{Si}$ at the $\mathrm{SiO}_{2} / \mathrm{Si}$ interface. In general, preferential etching can deteriorate or enhance the depth resolution as shown on a SiGe epilayer structure. Finally, the capabilities of zero-energy SIMS are shown on an ultra-shallow SiGe structure demonstrating a narrow surface transient and a very good depth resolution in the surface region. Copyright (c) 2010 John Wiley \& Sons, Ltd.

Keywords: Zero-energy SIMS; electron beam-induced etching; roughness development; preferential etching; depth resolution

\section{Introduction}

Owing to the scaling of semiconductor devices to the (sub-) 32-nm-technology, there is an increasing interest to study quantitatively ultra-shallow junctions and dopant profiles across interfaces with high depth (subnanometer) and high spatial (nanometer) resolution. Until now, SIMS still has a leading role due to its high sensitivity, quantification accuracy and depth resolution. However, these objectives become limited by the fundamental SIMS physics, i.e. the primary beam-induced collision cascade and the primary ion incorporation during the sputtering process. Lowering the energy of the primary beam to extremely low energies $(100-200 \mathrm{eV})$ reduces the primary beam penetration depth but cannot completely exclude the atomic mixing process. ${ }^{[1]}$ Therefore, other compositional analysis techniques like 3D Tomographic Atomprobe ${ }^{[2,3]}$ and zeroenergy SIMS ${ }^{[4,5]}$ are under investigation. Their material emission mechanisms are based on (laser-assisted) electric field evaporation and electron beam-induced etching (EBIE), respectively, and therefore, they completely remove the depth resolution limitations induced by the ion beam sputtering process. In particular, the goal of the zero-energy SIMS analysis technique is a localized material removal based on the interaction of an electron beam with the adsorbed reactive precursor molecules on the sample surface. This will stimulate the creation of a volatile compound between the precursor molecules and the matrix elements and therefore may, theoretically, lead to monolayer depth resolution. A detailed description of zero-energy SIMS can be found in Refs [4] and [6] and initial depth profiles on silicon with EBIE using xenon difluoride $\left(\mathrm{XeF}_{2}\right)$ as reactive precursor molecule are already reported ${ }^{[5]}$ with a depth resolution ( $1.5 \mathrm{~nm} / \mathrm{dec}$ ) commensurately to SIMS. To achieve its intrinsic depth resolution $(<0.5 \mathrm{~nm} / \mathrm{dec})$, a constant (almost) atomically flat surface during the etching process/depth profiling is crucial. However, spontaneous etching of silicon and SiGe with $\mathrm{XeF}_{2}$ is a highly isotropic etch process in which roughening of the surface is a major concern, whereas, $\mathrm{SiO}_{2}$ and $\mathrm{Si}_{3} \mathrm{~N}_{4}$ are only etched by $\mathrm{XeF}_{2}$ with the assistance of ion or electron irradiation. ${ }^{[7,8]}$

In this article, the roughness of surfaces etched with EBIE and $\mathrm{XeF}_{2}$ will be investigated and compared with the case of spontaneous etching. Owing to the activated enhancement of the etching process by irradiation of the surface with electrons, it is shown already that multiple reaction products $\left(\mathrm{SiF}_{x}, \mathrm{x}=0-4\right)$ are formed in contrast to only $\mathrm{SiF}_{4}$ as volatile reaction product during spontaneous etching of $\mathrm{Si}$ at room temperature. ${ }^{[5]}$ These differences in Si etch kinetics by EBIE can have an influence on the evolution of surface roughness. Also, different initial surface conditions like $\mathrm{H}$-terminated, native oxide and a thin thermal oxide will be investigated in order to reveal their influence on the roughness evolution and find the optimal conditions to perform zero-energy SIMS depth profiling. Finally, the influence of a multilayer system (SiGe epilayers) and preferential etching on the depth resolution will be examined.

\section{Experimental}

The zero-energy SIMS experiments were carried out in a conventional SIMS tool (Cameca IMS $5 f$ ) based on a magnetic sector mass spectrometer which is adapted for EBIE experiments. Different from the system described in ${ }^{[5]}$ which could only generate

\footnotetext{
Correspondence to: N. Vanhove, IMEC vzW, Kapeldreef 75, B-3001 Leuven, Belgium. E-mail:Nico.Vanhove@imec.be

a IMEC vzw, Kapeldreef 75, B-3001 Leuven, Belgium

b Instituut voor Kern- en Stralingsfysica, K.U. Leuven, Celestijnenlaan 200 D, B-3001 Leuven, Belgium

c Laboratorium voor Vaste-Stoffysica en Magnetisme, K.U. Leuven, Celestijnenlaan 200 D, B-3001 Leuven, Belgium
} 
a very broad beam, the electron beam is now mounted on the primary column (Cs-flange). Using the standard ion optics a focused electron beam of $9 \mathrm{keV}$ can then be obtained with a FWHM of approximately $50 \mu \mathrm{m}$ in spot mode and a current density of $25 \mathrm{~mA} / \mathrm{cm}^{2}$. With this configuration, it is possible to raster the focused electron beam and create a very good defined etch crater of $250 \times 250 \mu \mathrm{m}^{2}$. All the SIMS data are obtained in positive mode (positive sample potential) by electron beam impact ionization in the gas phase and the formation of cations on ionic surfaces (like the $\mathrm{SiF}_{X}$ reaction layer) by the electron stimulated desorption (ESD) process based on the Knotek-Feibelman mechanism. ${ }^{\left[{ }^{[]}\right.} \mathrm{XeF}_{2}$ was introduced as etching gas through a gas nozzle which is situated approximately $5 \mathrm{~mm}$ above the substrate. The corresponding partial $\mathrm{XeF}_{2}$ pressure in the chamber during the EBIE experiments was around $10^{-6}$ mbar. In previous work, ${ }^{[5]}$ the kinetic energy distribution (KED) of silicon cations shows still a small contribution of high-energy secondary ions (Thompson distribution). However, due to the new electron gun configuration with a lower current density and a slightly decreased partial pressure of $\mathrm{XeF}_{2}$, the sputter component in the silicon KED (not shown) is completely removed and the emission mechanisms are now fully based on ESD phenomena. All experiments are performed at room temperature.

\section{Results and Discussion}

To investigate the influence of EBIE on surface morphology, a comparison is made between a region on a Si substrate with and without electron beam exposure in the presence of $\mathrm{XeF}_{2}$ vapor. Therefore, a $n$-type $\mathrm{Si}(100)$ sample was prepared by removing the native oxide by dipping in a $2 \%$ hydrofluoric acid (HF) solution for $2 \mathrm{~min}$. The exposure time in $\mathrm{XeF}_{2}$ vapor was $135 \mathrm{~min}$. and the Si EBIE crater size was $250 \times 250 \mu \mathrm{m}^{2}$ with a $170 \mathrm{nA}$ electron beam. As a result, two etch depths are obtained whereby the most shallow one $(100 \mathrm{~nm})$ represents the spontaneous etching process of $\mathrm{XeF}_{2}$ without electron beam exposure and a Si EBIE crater of $208 \mathrm{~nm}$ which is deeper due to the enhanced $(2 \times)$ etch rate. Before etching, the initial root-mean-square (RMS) roughness measured with atomic force microscopy (Veeco Nanoscope IV AFM Dimension 3100) in tapping mode was around $0.2 \mathrm{~nm}$ (scan size: $2 \times 2 \mu \mathrm{m}^{2}$ ). After $\mathrm{XeF}_{2}$ exposure, AFM measurements are performed in the Si EBIE crater and in the proximity of the crater where the roughness is induced by spontaneous etching. A typical AFM cross section inside the EBIE crater is shown in Fig. 1. The obtained RMS roughness for EBIE and spontaneous etching of $\mathrm{Si}$ was 6.2 and $7.5 \mathrm{~nm}$, respectively.

By the spontaneous etching process of $\mathrm{Si}$, the main reaction mechanism is the subsequent attachment of $\mathrm{F}$ atoms to the $\mathrm{SiF}_{x}$ reaction layer to form $\mathrm{SiF}_{4}$ as volatile reaction product. Since $\mathrm{SiF}_{4}$ is a volatile product, it is not a significant component in the reaction layer but has to be formed in that layer. V. S. Aliev et al. proposed that the formation of $\mathrm{SiF}_{2}$ and $\mathrm{SiF}_{3}$ in the reaction layer is a nonactivated process (not surface site dependant) and, therefore, Si atoms are removed from random sites which induce the observed surface roughness development. ${ }^{[11]}$ However, the electron beam can change the thickness and structure of the reaction layer and therefore also influence the etch kinetics and roughness development. In the case of fluorine containing precursor molecules, fluorine can penetrate into the silicon lattice because no steric hindrance is present as observed with larger atoms as $\mathrm{Cl}$ and $\mathrm{Br}$ and exist primarily as negative ions. ${ }^{[10]}$ In the mass transport limited Si EBIE regime, where the electron

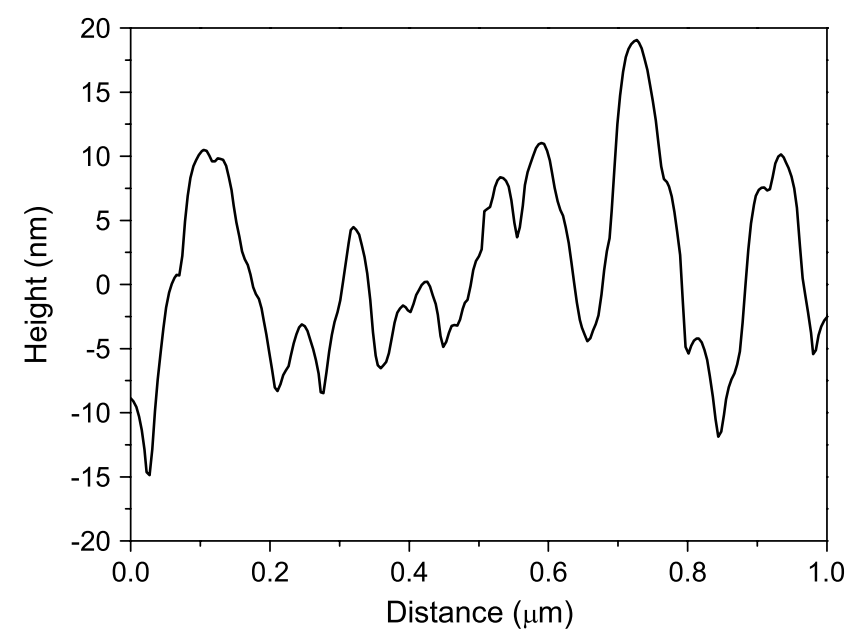

Figure 1. Typical AFM cross-section of a Si substrate after electron beaminduced etching with $\mathrm{XeF}_{2}$.

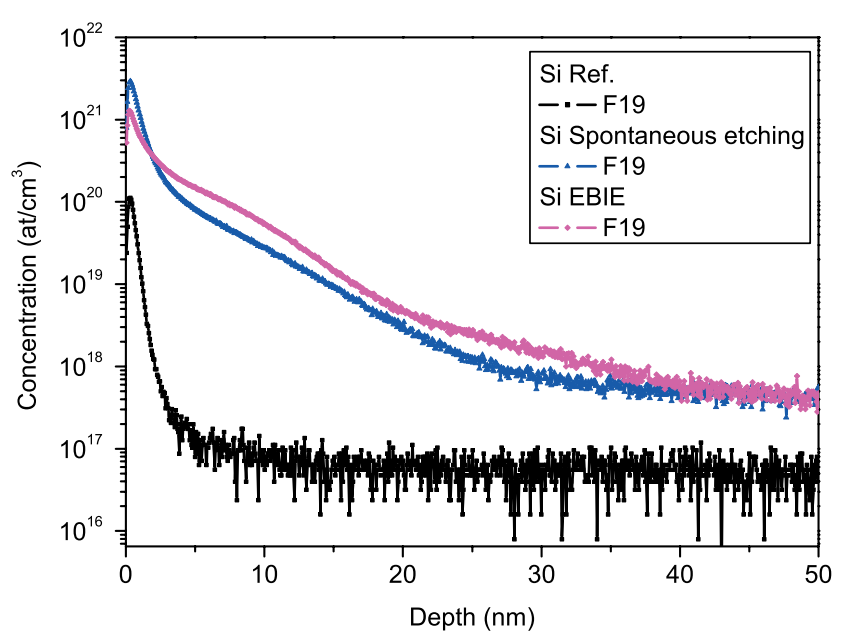

Figure 2. Concentration of $\mathrm{F}$ as function of the depth for a Si sample with spontaneous etching in a $\mathrm{XeF}_{2}$ vapor and with Si EBIE. As reference the depth profile of the always apparent $F$ surface peak is shown.

flux is much greater than the impingement rate of the precursor molecules, one expects that the surface concentration of the precursor would be essentially zero due to the dominating desorption. In this case, the thickness of the reaction layer and the (nonactivated) formation of the $\mathrm{SiF}_{x}$ components can be reduced. Ideally, the reaction layer has to be limited to only one $\mathrm{Si}-\mathrm{F}$ monolayer to reduce the surface roughness. However, a corresponding SIMS profile ( $500 \mathrm{eV} \mathrm{Cs,} \mathrm{Atomika} \mathrm{4500)} \mathrm{shows}$ a dilute concentration of fluorine extending over more than $20 \mathrm{~nm}$ in the lattice (Fig. 2) similar to the distribution by a spontaneous etching process. Corresponding to the observed lower $\mathrm{F}$ concentration in the reaction layer and the intensity distribution of the different $\mathrm{SiF}_{X}$ species, ${ }^{[5]}$ it seems that the $\mathrm{SiF}_{X}$ species distribution is changed from a more pronounced $\mathrm{SiF}_{2}-\mathrm{SiF}_{3}$ species coverage by spontaneous etching with $\mathrm{XeF}_{2}$ to a surface layer where the contribution of SiF is dominant. This subsurface fluorine concentration can still have a large influence on the (nonactivated) etch kinetics and, therefore, by the random nature of $\mathrm{SiF}_{x}$ compound formation on the surface roughness development. 


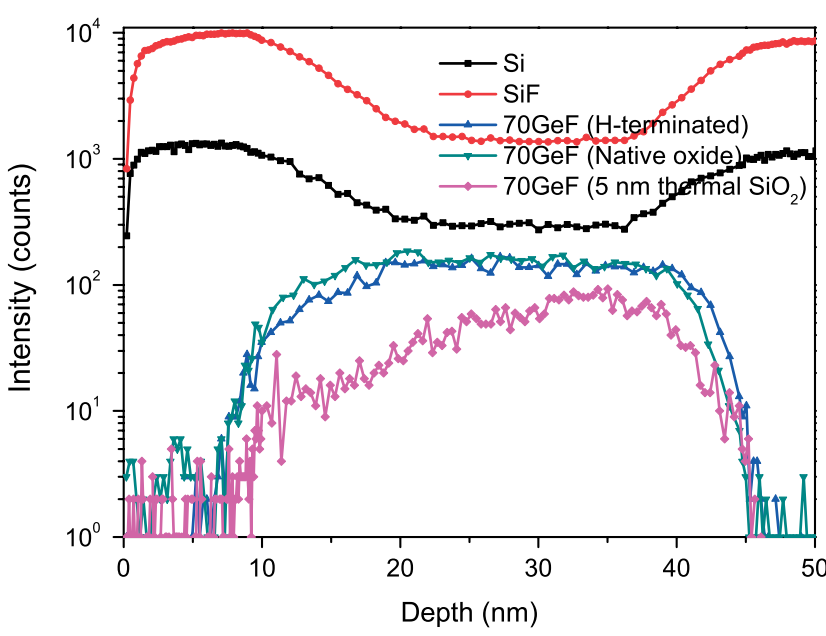

Figure 3. Zero-energy SIMS depth profile of a $35 \mathrm{~nm}$ SiGe epilayer with a $10 \mathrm{~nm} \mathrm{Si}$ cap layer with different top layers (H-terminated, native oxide and thermal $\mathrm{SiO}_{2}$ ). For the $\mathrm{H}$-terminated sample, also the $\mathrm{Si}$ and $\mathrm{SiF}$ signals are shown.

Until now, the influence of the different reaction mechanisms on the surface morphology is discussed for EBIE in comparison with spontaneous etching. However, the initial surface conditions and the presence of multilayer structures can also have a large influence on the initial reaction of $\mathrm{F}$ with $\mathrm{Si}$ and on the etch process and roughness development on a larger timescale. Previously, the native oxide was used to prevent the spontaneous etching of $\mathrm{Si}$ in a $\mathrm{XeF}_{2}$ vapor but (mechanically induced) damage to this thin oxide can create small regions where spontaneous etching directly starts. Therefore, an approx. $5 \mathrm{~nm}$ thermal $\mathrm{SiO}_{2}$ layer $(15 \mathrm{~min}$ at $800^{\circ} \mathrm{C}$ in an $\mathrm{O}_{2}$ atmosphere) was grown on the samples to prevent spontaneous etching. To study also the influence of a multilayer system, a $35 \mathrm{~nm}$ strained SiGe (Ge content $=19 \%$ ) epilayer was grown on a silicon substrate with a $10 \mathrm{~nm}$ silicon cap layer on top. The zero-energy SIMS depth profiles for this sample with different top layers ( $5 \mathrm{~nm}$ thermal $\mathrm{SiO}_{2}$, native oxide and H-terminated) is shown in Fig. 3. For the H-terminated sample, also the $28 \mathrm{Si}$ and $47 \mathrm{SiF}$ signals are shown. It is clear that a thermal $\mathrm{SiO}_{2}$ top layer gives rise to a poor depth resolution. To explain this, an AFM cross-section of a small etch crater section is shown in Fig. 4 and shows the formation of extreme etch pitting during electron beam induced etching (up to $30 \mathrm{~nm}$ on a etch crater of $60 \mathrm{~nm}$ ). Pit formation was observed as well on a thick $\mathrm{SiO}_{2}$ layer, but on a smaller scale. We believe the pit formation is initiated by small defects in the $\mathrm{SiO}_{2}$ layer. These sites can form preferred adsorption sites for the precursor molecules and increase locally the etch rate. However, another mechanism has to be involved to explain this extreme pit formation. When the initial pit is formed in the thin thermal oxide layer and reach the $\mathrm{SiO}_{2} / \mathrm{Si}$ interface, the etch rate increases locally very rapidly. A ten times faster etch rate in a $\mathrm{Si}(100)$ substrate in comparison to a thermal $\mathrm{SiO}_{2}$ layer is observed due to the combined spontaneous etching and the increased desorption of the $\mathrm{SiF}_{x}$ reaction products in the Si layer. At the interface the roughness layer accumulates when etching the thermal $\mathrm{SiO}_{2}$ layer and the created roughness is proportional to the thickness of this layer. Therefore the duration of this preferential etching determines the absolute roughness thickness at the end of the $\mathrm{SiO}_{2}$ EBIE. This mechanism leads to high-aspect-ratio etches pits where also the diameter can increase during EBIE by the increase of surface sites on the wall where etching can occur. Due

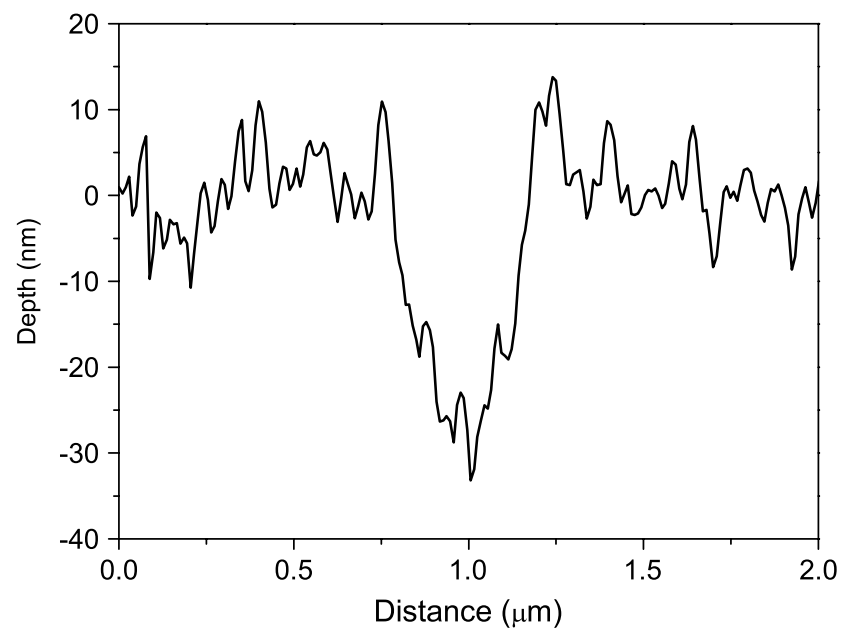

Figure 4. AFM cross-section of the high-aspect-ratio etch pits formed by $\mathrm{SiO}_{2}$ EBIE and preferential etching at the $\mathrm{SiO}_{2} / \mathrm{Si}$ interface.

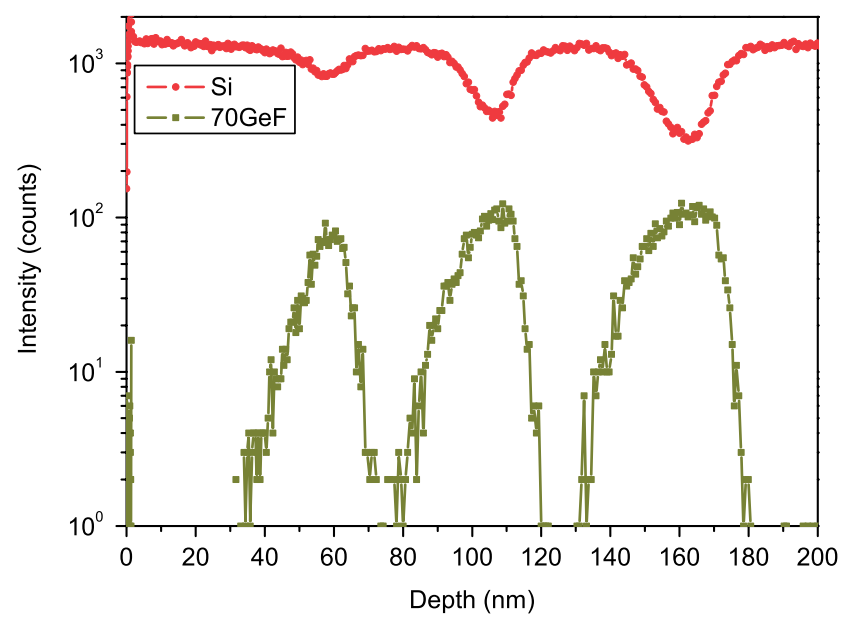

Figure 5. Zero-energy depth profile of a SiGe stack (same Ge content: $20 \mathrm{~nm}-30 \mathrm{~nm}-40 \mathrm{~nm}$ ) with the native oxide as top layer.

to the thin native oxide layer $(10-15 \AA)$, these high-aspect-ratio etch pits cannot be formed and, therefore, the depth resolution is much better up to $2.5 \mathrm{~nm} / \mathrm{dec}$ for the leading slope. A comparable depth resolution is achieved for the $\mathrm{H}$-terminated surface where a comparable mechanism can play a role. The fact that a $\mathrm{H}$-terminated surface is an energetically more stable surface than a F-terminated one, may induce a slower $\mathrm{H}$ removal process before regular etching can start by the buildup of a $\mathrm{SiF}_{X}$ reaction layer.

In general, preferential EBIE etching at the interface of the bottom layer over the top layer results in a fast roughening and deterioration of the depth resolution while preferential etching of the top layer over the bottom layer can have a polishing effect and results in an increased depth resolution. An example is given in Fig. 5 where a depth profile is shown of a SiGe epilayer stack with the native oxide as top layer. The depth resolution of the leading edge is around $10 \mathrm{~nm} / \mathrm{dec}$ while the trailing edge has a much better depth resolution of about $3.5 \mathrm{~nm} / \mathrm{dec}$. This can be explained by the difference in EBIE etch rate for SiGe (Ge content $=20 \%$ ) which is 3 times faster than for Si. This ratio may be dependant on Ge concentration and strain in the SiGe epilayer. This induces an increased roughness layer thickness at the Si/SiGe 


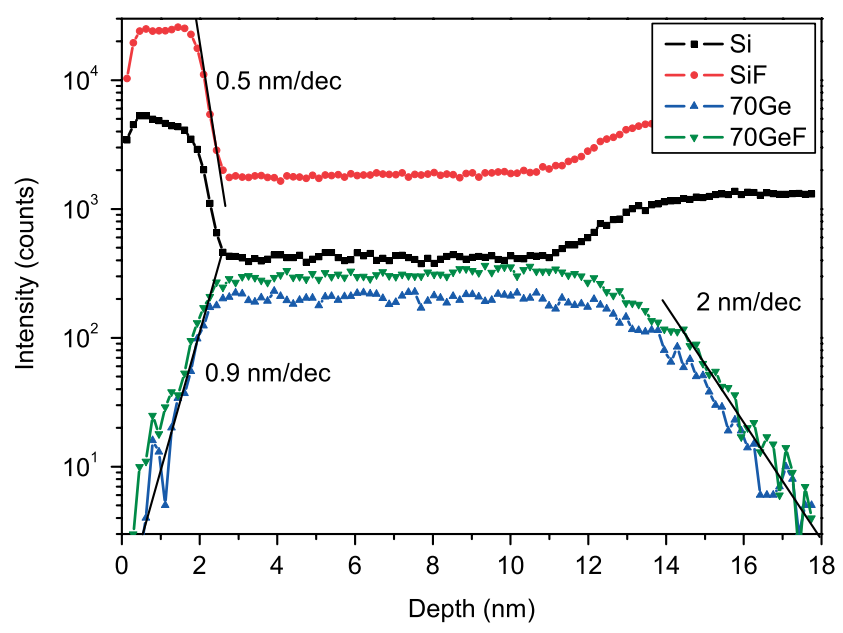

Figure 6. Zero-energy SIMS depth profile of a $10 \mathrm{~nm}$ SiGe epilayer (Ge content $=45 \%$ ) with a $2 \mathrm{~nm}$ Si layer on top. A HF-dip was done before the measurement.

interface (leading edge) while a polishing effect appears at the $\mathrm{SiGe/Si} \mathrm{interface} \mathrm{(trailing} \mathrm{edge).} \mathrm{Despite} \mathrm{these} \mathrm{limiting} \mathrm{factors} \mathrm{due}$ to roughness development by Si EBIE with $\mathrm{XeF}_{2}$, the capabilities of Zero-energy SIMS are shown in Fig. 6 for a $10 \mathrm{~nm} \mathrm{SiGe} \mathrm{(Ge} \mathrm{content}$ $=45 \%$ ) epilayer grown on a Si substrate with a $2 \mathrm{~nm}$ Si cap layer. For the Si and SiF signals, an extremely high depth resolution of $0.5 \mathrm{~nm} / \mathrm{dec}$ at the $\mathrm{Si} / \mathrm{SiGe}$ interface in the surface region can be obtained. These signals drop by one order of magnitude at the interface while only a drop of a factor two is expected in the case of quantitative depth profiles. These large changes in the distribution of the different reaction products $\left(\mathrm{SiF}_{x}, \mathrm{GeF}_{x}, \mathrm{SiGeF}_{x}\right.$ ?? ...) in the different layers can be caused by an abrupt change in the composition and morphology of the EBIE reaction layer. Due to the difference in Si and SiGe EBIE rates, the obtained depth resolution at the interface can be also influenced by these facts. Therefore, the leading slope of the Ge signal can give more useful information. Here, a depth resolution of $0.9 \mathrm{~nm} / \mathrm{dec}$ can be obtained which is comparable with an extremely low-energy (150 eV O 2 beam) SIMS depth profile (not shown). At the SiGe/Si interface the depth resolution is decreased to $2 \mathrm{~nm} / \mathrm{dec}$ due to roughness development by the random removal of atoms in the EBIE process.

\section{Conclusion}

To achieve monolayer resolution by the zero-energy SIMS technique, a controlled EBIE process is crucial. At the moment, a focused electron beam is implemented and the sputter component due to unwanted gas phase reactions is completely removed in the kinetic energy distribution of silicon and therefore shows that the new concept can be implemented in a magnetic sector SIMS with a high extraction field. However, Si EBIE in a $\mathrm{XeF}_{2}$ vapor shows the same surface roughness development as has been observed by the spontaneous reaction. Due to steric effects with fluorine-based precursor molecules, a dilute concentration of fluorine is observed in the substrate up to $20 \mathrm{~nm}$ even in the masslimited Si EBIE regime which can have a large influence on etch kinetics. However, different precursor molecules and experimental conditions have to be explored to minimize the surface roughness. Owing to these created roughness, preferential etching at an interface (etch rate $\mathrm{Si}=10 \times \mathrm{SiO}_{2}$ and etchrate $\mathrm{SiGe}=3 \times \mathrm{Si}$ ) can drastically influence the depth resolution at the leading and trailing edge due to an increased roughness development or a polishing effect. However, due to a narrow surface transient, a high depth resolution $(<1 \mathrm{~nm} / \mathrm{dec})$ is demonstrated in the surface region.

\section{References}

[1] W. Vandervorst, Appl. Surf. Sci. 2008, 255, 805.

[2] Thomas F. Kelly, Michael K. Miller, Rev. Sci. Instrum. 2007, 78, 031101.

[3] S. Koelling, W. Vandervorst, (these proceedings).

[4] N. Vanhove, P. Lievens, W. Vandervorst, Appl. Surf. Sci. 2008, 255, 1360.

[5] N. Vanhove, P. Lievens, W. Vandervorst, Phys. Rev. B 2009, 79, 035305.

[6] W. Vandervorst, US Patent, No. 20,030,127,591.

[7] H. F. Winters, J. W. Coburn, Appl. Phys. Lett. 1979, 34, 70.

[8] G. Xuan T. N. Adam, P.-C. Lv, N. Sustersic, M. J. Coppinger, J. Kolodzey, J. Suehle, E. Fitzgerald, J. Vac. Sci. Technol. A 2008, $26,385$.

[9] M. L. Knotek, P. J. Feibelman, Phys. Rev. Lett. 1978, 40, 964.

[10] H. F. Winters, D. B. Graves, D. Humbird, S. Tougaard, J. Vac. Sci. Technol. A 2007, 25, 96.

[11] V. S. Aliev, V. N. Kruchinin, Surf. Sci. 1999, 442, 206. 